\title{
The First Case of a Staphylococcus pseudintermedius Infection after Joint Prosthesis Implantation in a Dog
}

\author{
JACEK MIĘDZOBRODZKI ${ }^{1 *}$, ANDRZEJ KASPROWICZ ${ }^{2}$, ANNA BIAŁECKA ${ }^{2}$, OLGA JAWORSKA $^{3}$, \\ KLAUDIA POLAKOWSKA ${ }^{1}$, BENEDYKT WŁADYKA ${ }^{1}$ and ADAM DUBIN ${ }^{1}$ \\ ${ }^{1}$ Faculty of Biochemistry, Biophysics and Biotechnology, Jagiellonian University, Kraków, Poland \\ ${ }^{2}$ Centre for Microbiological Research and Autovaccines, Kraków, Poland \\ ${ }^{3}$ Veterinary Clinic "Alvet", Kraków, Poland
}

Received 6 February 2010, revised 8 March 2010, accepted 20 March 2010

\begin{abstract}
We have reported a bacterial infection in a dog with progressive dysplasia of the hips. Orthopedic surgery was performed. Seven weeks prior to the surgery, the patient was bitten by another dog. The postimplantation wound exuded for four days after the surgery. Microbiological analysis performed by standard identification techniques showed the presence of Staphylococcus intermedius, but an additional molecular analysis indicated S. pseudintermedius. This was followed by an evaluation of antibiotic susceptibility of the strain which showed cefoxitin, ciprofloxacin, clindamycin, trimethoprim/sulfamethoxazole, doksycycline, erythromycin, and gentamicin resistance. Minimal inhibitory concentration (MIC) values for selected antibiotics were reported. Resistance for cefoxitin indicates that methicillin resistant S. pseudintermedius (MRSP) strains were present in individual macroorganisms, but they can expand and persist the colonization of other hosts.
\end{abstract}

Key words: Staphylococcus pseudintermedius, infection, molecular diagnostics, dog

Description of the case. The host of the bacterial infection was a seven year old, non-castrated, Bergamasco Sheepdog weighing $40 \mathrm{~kg}$ before surgery. For the past 4-5 years the dog displayed limping in the pelvic limb. Clinical examination of the dog revealed considerable crepitation. The dog had difficulty standing up after prolonged rest and displayed increasing aversion to movement. Diagnostic radiography showed progressive dysplasia of the right hip and dysplasia of the left hip with degenerative changes. The patient was sent to an orthopedic surgeon who recommended prosthetic joint replacement surgery. Each implant procedure involved the removal of the damaged joint and an artificial prosthesis replacement. Moreover, the owner of the dog reported that the dog had been bitten by a large five year old mixedbreed dog. After the bite the wound was cleaned and disinfected with the use of antiseptic substances, and also prophylactically ceftriaxon with metronidazole were applied. The incident occurred seven weeks prior to the surgery in the region of the right trochanster major femoral bone (distal to the area of the surgery). Four days after the implantation surgery, the wound began to exude. At the beginning the volume of the discharge was about 5-8 $\mathrm{ml}$ a day, later it increased to about $30 \mathrm{ml}$. The dog had a fever and was apathetic, sore and did not eat. The wound healed up after a onemonth treatment with linezolid. Three months after the surgery the prosthesis was removed. During the healing process three samples for microbiological analysis were taken. The first sample was taken from the seeping wound one week after the surgery. The second sample originated from the marrow cavity of the femoral bone during the removal of the prosthesis. The third sample was taken from the skin around the scar after the healing process.

Microbiological diagnostics. Identification. Each sample was cultured in a blood agar medium (TSA $+5 \%$ of sheep blood), in a selective medium for staphylococci (SAID, BioMerieux), and in a medium for rods (CPS, BioMerieux). The obtained cultures observed by microscopic testing demonstrated the presence of staphylococci which were then further analyzed by a free coagulase tube test and by an identification system for staphylococci ID32 STAPH (BioMerieux). The bacteria were identified as S. intermedius. However,

\footnotetext{
* Corresponding author: J. Międzobrodzki, Department of Microbiology, Faculty of Biochemistry, Biophysics and Biotechnology, Jagiellonian University, Gronostajowa Str. 7, 30-387 Kraków, Poland; phone: (+48) 12 6646371; e-mail: jacek.miedzobrodzki@uj.edu.pl
} 
from our experience, the ID32 STAPH identification system may give ambiguous results for isolates of animal origin. Moreover the test does not include a recently described coagulase-positive species $-S$. pseudintermedius (Devriese et al., 2005). Therefore final identification of the isolates was performed using molecular analysis (Bannoehr et al., 2009; Black et al., 2009). Among methods, including PCR-RFLP of glyceraldehyde-3-phosphate dehydrogenase-encoding gene, sequencing of internal fragments of the $16 \mathrm{SrRNA}$ and $r p o B$ genes, used for $S$. pseudintermedius identification only the last is discriminatory (Władyka et al., 2008). For partial sequence analysis of the $r p o B$ gene, PCR was performed according to the protocol described by Drancourt and Raoult (Drancourt and Raoult, 2002). The obtained fragment was sequenced with the primers used for the amplification. All three isolates had an identical sequence of the rpoB gene. Comparison of the sequence with the corresponding ones of S. pseudintermedius strains deposited in the EMBL database revealed either their full identity or one nucleotide difference between the strains, whereas homo$\operatorname{logy}$ with $S$. intermedius was only $95 \%$. Therefore the isolates were finally identified as $S$. pseudintermedius. The sequence for the partial $r p o B$ gene was deposited in the EMBL database under accession number FN556020.

Antimicrobial susceptibility testing. Evaluation of antibiotic susceptibility of the isolates was performed by a disc-diffusion test, and an E-test (AB BIODISK) according to the guidelines set by the National Committee for Clinical Laboratory Standards (NCCLS), (Faires et al., 2009; Sasaki et al., 2007). The disc diffusion method with cefoxitin was used to analyze the methicillin resistance (Swenson et al., 2005). Additionally two discs, with erythromycin and clindamycin, were used to examine resistance to macrolides, linkosamids, and streptogramins B. The methicillin resistant $S$. pseudintermedius also showed resistance for macrolides, lincosamids and streptogramins $\mathrm{B}$, implicating the constitutive mechanism (MLS ${ }_{\mathrm{B}}$ constitutive). This was found in each of the taken samples. The results of antimicrobial testing are presented in Table I. The MIC values achieved from the E-tests performed for selected antibiotics are reported too.

The presence of a chromosomal cassette SCCmec type III encoding antibiotic multiresistance was confirmed using MULTIPLEX PCR analysis, which is a useful tool used for the identification of structural types and variants of the mec gene in staphylococci. Additionally, the isolates analyzed were compared to the standard strains (Oliveira and deLancastre, 2002).

Comments and conclusions. Accurate identification of bacteria from the genus Staphylococcus isolated from animals or men is important from a laboratory diagnostics point of view, as well as in clinical practice. Some staphylococcal species are reported more
Table I

Antibiotic susceptibility of the Staphylococcus pseudintermedius strain

\begin{tabular}{|l|c|c|c|}
\hline \multicolumn{1}{|c|}{ Antibiotic } & $\begin{array}{c}\text { Diameter of the } \\
\text { inhibition zone } \\
(\mathrm{mm})\end{array}$ & MIC & Result \\
\hline Cefoxitin & 6 & - & resistant \\
\hline Chloramphenicol & 18 & $2.0 \mathrm{mg} / 1$ & sensitive \\
\hline Ciprofloxacin & 6 & - & resistant \\
\hline Clindamycin & 6 & - & resistant \\
\hline $\begin{array}{l}\text { Trimethoprim/ } \\
\text { Sulfametoxazole }\end{array}$ & 6 & - & resistant \\
\hline Doksycycline & 6 & - & resistant \\
\hline Erythromycin* & 6 & - & resistant \\
\hline Fusidic acid & 26 & - & sensitive \\
\hline Gentamicin & 6 & - & resistant \\
\hline Linezolid & 23 & $0.5 \mathrm{mg} / 1$ & sensitive \\
\hline Mupirocin & 30 & - & sensitive \\
\hline Rifampicin & 21 & $0.002 \mathrm{mg} / 1$ & sensitive \\
\hline Teicoplanin** & - & $0.5 \mathrm{mg} / 1$ & sensitive \\
\hline Vancomycin** & - & $1.5 \mathrm{mg} / 1$ & sensitive \\
\hline
\end{tabular}

* The result for erythromycin susceptibility is also representative for roxithromycin, clarithromycin, azithromycin, and dirithromycin.

** The diameter of the inhibition zone was not tested by the disc diffusion method.

frequently than others in samples from infections, and exhibit an increasing level of antibiotic resistance. Many staphylococcal species belong to the normal biocenosis, however under favorable conditions the bacteria may cause serious infections. In the case described above we performed molecular analysis followed after the identification based on the biochemical characteristic of the pathogen isolated from the infection after the implantation of the prosthesis. The presence of $S$. intermedius, an opportunistic species participating in normal physiological biocenosis of healthy dogs was reported in routine diagnostic procedures (Hauschild and Wójcik, 2007). However the surgical intervention was the additional factor which favored bacteria to increase infection. Unfortunately, it is unknown if the strain was present in the skin before the bite or if it was introduced by the dog aggressor?

The samples for bacteriological examination were taken three times during the therapy process. From the first sample a rich culture of $S$. intermedius was achieved despite prior application of the antibiotic (ceftriaxon and metronidazole) to the patient. Based on the above observation, and on the antibiogram, linezolid was administrated to the dog (7.5 $\mathrm{mg}$ per 1 $\mathrm{kg}$ of the body weight). Then from the second and the third samples only single Staphylococcus intermedius colonies were grown. All the isolates exhibited the same antibiotic resistance pattern.

Examinations of the S. intermedius group (SIG) show new challenges in diagnostics therefore the 
selection of techniques and tests for phenotypic and genetic analysis are in trial phases, so any information on their properties is very valuable (KizerwetterŚwida et al., 2009). Phenotypic typing methods used in standard diagnostic tests were not effective to discriminate $S$. pseudintermedius from $S$. intermedius. Therefore, analysis of the partial rpoB gene sequence of the isolates was performed. The results showed that the isolates examined were identical and were classified as S.pseudintermedius. According to recent guidelines, strains isolated from dogs that are identified by standard biochemical tests as $S$. intermedius should be reported as S. pseudintermedius, and generally there is a need to elaborate the regulation system when classifying the SIG group (Devriese et al., 2009; Ruscher et al., 2009).

Due to surgery and adequate chemotherapy based on the antibiogram, the therapeutic process was concluded successfully. The antibiogram indicated effective antibacterial medicines. Surprisingly, the examined isolate showed multi drug resistance, particularly for $\beta$-lactams, including methicillin, allowing classification of the strain as a methicillin resistant $S$. pseudintermedius (MRSP).

Temporary colonization of man by animal strains, the horizontal transfer of genes, and the antibiotic resistance, including methicillin-resistance among animal isolates cause an alarming phenomenon which could result in dangerous clinical consequences in the future (Black et al., 2009; Weese and Van Duikeren, 2009).

In conclusion, we described the isolation of a methicillin resistant $S$. pseudintermedius (MRSP) which was the etiological factor of a wound infection in the dog which was surgically operated because of degenerative changes, but prior to the operation was bitten by another dog. This confirms the dangerous dissemination of drug resistance in staphylococci strains of animal origin. Phenomena of temporary colonization and carriage of such strains by dog owners, together with reports on S. pseudintermedius infections among humans (VanHoovels et al., 2006), led us to conclude that novel strains carrying antibiotic resistant genes will soon be reported. Thus, the establishment of new procedures and diagnostic schemes for both veterinary and human microbiology are an emerging challenge.

\section{Acknowledgements}

The authors would like to thank Miss Małgorzata Wójcik for helping prepare this manuscript. The project was partially supported by the grant no N302 130734 from the Polish Ministry of Science and Higher Education (MNiSW).

\section{Literature}

Bannoehr J., A. Franco, M. Iurescia, A. Battisti and R. Fitzgerald. 2009. Molecular Diagnostic Identification of Staphylococcus pseudintermedius. J. Clin. Microbiol. 2: 469-471.

Black C.C., S.M. Solyman, L.C. Eberlein, D.A. Bemis, A.M. Woron and S.A. Kania. 2009. Identification of predominant multilocus type, pulsed-field gel electrophoresis cluster, and novel staphylococcal chromosomal cassette in clinical isolates of mecA-containing, methicillin resistant Staphylococcus pseudintermedius. Vet. Microbiol. 139: 333-338.

Devriese L.A., K. Hermans, M. Baele and F. Haesebrouk. 2009. Staphylococcus pseudintermedius versus Staphylococcus intermedius. Vet. Microbiol. 133: 206-207.

Devriese L.A., M. Vancanneyt, M. Baele, M.Vaneechoutte, E. De Graef, C. Snauwaert, I. Cleenwerck, P. Dawyndt, J. Swings, A. Decostere and F. Haesebrouck. 2005. Staphylococcus pseudintermedius sp. nov., a coagulase-positive species from animals. Int. J. Syst. Evol. Microbiol. 55: 1569-1573.

Drancourt M. and D. Raoult. 2002. rpoB gene sequence-based identification of Staphylococcus species. J. Clin. Microbiol. 40: 1333-1338.

Faires M.C., S. Gard, D. Aucoin and J.S. Wees. 2009. Inducible clindamycin-resistance in methicillin resistant Staphylococcus aureus and methicillin-resistant Staphylococcus pseudintermedius isolates from dogs and cats. Vet. Microbiol. 139: 419-420.

Hauschild T. and A. Wójcik. 2007. Species distribution and properties of staphylococci from canine dermatitis. Res. Vet. Sci. 82: $1-6$.

Kizerwetter-Świda M., D. Chrobak, M. Rzewuska and M. Binek. 2009. Antibiotic resistance patterns and occurrence of mecA gene in Staphylococcus intermedius strains of canine origin. Pol. J. Vet. Sci. 1: 9-13.

Oliveira D.C. and H. deLancastre. 2002. Multiplex PCR strategy for rapid identification of structural types and variants of the mec element in methicillin-resistant Staphylococcus aureus. Antimicrob. Agents Chemother. 7: 2155-2161.

Ruscher C., A. Lubke-Becker, C.G. Wleklinski, A. Soba, L.H. Wieler and B. Walther. 2009. Prevalence of methicillinresistant Staphylococcus pseudintermedius isolated from clinical samples of companion animals and equidaes. Vet. Microbiol. 136: 197-201.

Sasaki T., K. Kikuchi, Y. Tanaka, N. Takahashi, S. Kamata and K. Hiramatsu. 2007. Methicillin-resistant Staphylococcus pseudintermedius in a veterinary teaching hospital. J. Clin. Microbiol. 4: 1118-1125.

Swenson J.M., Tenover F.C. and Cefoxitin Disc Study Group. 2005. Results of disc diffusion testing with cefoxitin correlate with presence of mecA on Staphylococcus spp. J. Clin. Microbiol. 43: 3818-3823.

VanHoovels L., A. Vankeerberghen, A. Boel, K. VanVaerenbergh and H. DeBeenhouwer. 2006. First Case of Staphylococcus pseudintermedius Infection in a Human. J. Clin. Microbiol. 12: 4609-4612.

Weese J.S. and E. Van Duikeren. 2009. Methicillin-resistant Staphylococcus aureus and Staphylococcus pseudintermedius in veterinary medicine. Vet. Microbiol. doi:10,1016/j.vet.mic.2009.01.039. Wladyka B., M. Biśta, A.J. Sabat, E. Bonar, S. Grzeszczuk, W. Hryniewicz and A. Dubin. 2008. A Novel Member of the Thermolysin Family; Cloning and Biochemical Characterization of Metalloprotease from Staphylococcus pseudintermedius. Acta Biochim. Pol. 3: 523-536. 